\title{
Digital Video Watermarking Using Dwt And Pca
}

\author{
Supriya A. Patil ${ }^{1}$, Prof. Navin Srivastava ${ }^{2}$ \\ ${ }^{I} M$ Tech Student Dept of Electronics Engineering, B.V.D.U.COE Pune, India \\ ${ }^{2}$ Prof. Dept of Electronics Engineering, B.V.D.U.COE Pune, India
}

\begin{abstract}
Digital Watermarking may be a technology used for the copyright protection of digital applications. During this paper, a comprehensive approach for watermarking digital video is introduced, and a hybrid digital video watermarking scheme based on Discrete Wavelet Transform (DWT) and Principal Component Analysis (PCA). PCA helps in reducing correlation among the wavelet coefficients obtained from wavelet decomposition of each video frame thereby dispersing the watermark bits into the uncorrelated coefficients. The video frames are first decompose using DWT and also the binary watermark is embedded in the principal components of the low frequency wavelet coefficients. An imperceptible high bit rate watermark embedded is robust against various attacks that will be carried out on the watermarked video, like filtering, geometric attacks and contrast adjustment.
\end{abstract}

Keywords: - Binary Watermark., Digital Video, Discrete Wavelet Transform, Inverse DWT, Inverse PCA, Principal Component Analysis.

\section{INTRODUCTION}

Watermarking is a concept of embedding special pattern into multimedia document so that given piece of information is permanently tied to the data[1]. It inserts authentication data such as ownership information and $\operatorname{logo}$ in the digital media without affecting its original quality. Authentication data can be used as proof of ownership[1,2].

Watermarking can be done for different types of digital data where copyright needs to be protected. Digital watermarks are used to verify the authenticity of the carrier signal. It is prominently used for tracing copyright violations. Like traditional watermarks, digital watermarks are only perceptible under certain conditions, i.e. after using some algorithm[3]. A watermark is a digital code permanently embedded into cover content, in case of this system, into a video sequence[4,5].

Video watermarking approaches will be classified in to two main classes. The two classes are: Spatial domain and Transform domain watermarking.Spatial domain watermarking wherever embedding and detection of watermark are performed by directly manipulating the pixel intensity values of the video frame. Transform domain techniques[8-9], on the totally different hand, alter spatial pixel values of the host video according to a pre-determined transform and are more robust than spatial domain techniques since they disperse the watermark in the spatial domain of the video frame making it tough to remove the watermark through malicious attacks like cropping, scaling, rotations and geometrical attacks. The commonly used transform domain techniques are Discrete Fourier Transform (DFT), the Discrete Cosine Transform (DCT), and the Discrete Wavelet Transform (DWT) [8-9].

\section{WATERMARKING SCHEME}

For implementation of robust video watermarking scheme following transforms are used. Discrete Wavelet Transform (DWT) Principle Component Analysis (PCA).

\subsection{DiscreteWaveletTransform(DWT)}

Image is represented as a two dimensional (2D) array of coefficients, every coefficient representing the brightness level at that point. Most natural images have smooth color variations, with the fine details being described as sharp edges in between the smooth variations. Technically, the smooth variations in color could also be termed as low frequency components and therefore the sharp variations as high frequency components. The low frequency components constitute base of an image, and also the high frequency components add upon them giving a detailed image. Hence, the averages/smooth variations are demanding a lot of importance than the details [imp DWT].

DWT is used to implement a simple watermarking scheme. The 2-D discrete wavelet transforms (DWT) decomposes the image into sub-images. The approximation look like the original, only on the $1 / 4$ scale. The 2-D DWT is an application of the 1-D DWT in both the horizontal and also the vertical directions. The DWT decompose an image into a lower resolution approximation image (LL) as well as horizontal (HL), 
vertical ( $\mathrm{LH})$ and diagonal $(\mathrm{HH})$ detail components.Due to its excellent spatial-frequency localization properties DWT is very suitable to identify areas in the host video frame where a watermark can be embedded imperceptibly. Embedding the watermark in low frequencies obtained by wavelet decomposition increases the robustness with respect to attacks that have low pass characteristics like lossy compression, filtering, and geometric distortions.

Video is nothing but collection of still images. Original video is converted into frames discrete wavelet transform (DWT) and principal component analysis (PCA) are applied on each frame. Watermark image is converted into vectors and embedded in the low frequency (LL) DWT sub-bands of each decomposed frame. Embedding the watermark in both LL and HH makes the scheme robust to a variety of low and high frequency characteristic attacks [1]. Then inverse DWT and inverse PCA is applied to get watermarked video. One frame is chosen from watermarked video for implementation.

\subsection{Principal Component Analysis}

In digital image processing field, PCA is considered as a linear transform technique to convey most information about the image to principal components. PCA is a method of identifying patterns in data, and expressing the data in such a way so as to highlight their similarities and differences. Once these patterns in the data have been identified, the data can be compressed by reducing the number of dimensions, without much loss of information. It plots the data into a new coordinate system where the data with maximum covariance are plotted together and is known as principal component.

PCA transform is used to embed the watermark in each colour channel of each frame of video. The main advantage of this approach is that the same or multi-watermark can be embedded into the three colour channels of the image in order to increase the robustness of the watermark.

\section{WATERMARK EMBEDDING AND EXTRACTING PROCESS}

\subsection{Watermark embedding process}

Here first video is divided into frames. Then luminance component of each frame is chosen and DWT is applied to it which results into different sub bands. These bands are again divided into different blocks on which PCA is applied. For each block covariance matrix is calculated. Then each block is transforms into PCA components. watermark image is taken. This vector $\mathrm{W}$ is divided into four parts $\mathrm{p} 1, \mathrm{p} 2, \mathrm{p} 3$, and $\mathrm{p} 4$. Then each part is embedded into each of the corresponding sub bands. Inverse PCA is applied on the modified sub bands to obtain the modified wavelet block. By applying the inverse DWT watermarked luminance component of the frames are obtained. Finally by reconstructing the watermarked frame watermarked video is obtained.

\subsection{Watermark Extraction Process}

Here first video is divided into frames, RGB frame is converted into YUV frames. Then luminance component of each frame is chosen and DWT is applied to it which results into different sub bands. These bands are again divided into different blocks on which PCA is applied. For each block covariance matrix is calculated. Then each block is transforms into PCA components. On the other hand RGB watermark image is converted into binary image. This binary image is converted into a vector of zeros and ones. This vector $\mathrm{W}$ is divided into four parts $\mathrm{p} 1, \mathrm{p} 2, \mathrm{p} 3$, and $\mathrm{p} 4$. Then each part is embedded into each of the corresponding sub bands. Inverse PCA is applied on the modified sub bands to obtain the modified wavelet block. By applying the inverse DWT watermarked luminance component of the frames are obtained. Finally by reconstructing the RGB watermarked frame watermarked video is obtained.

\section{RESULT AND ANALYSIS}

\subsection{Peak Signal to Noise Ratio (PSNR)}

The Peak-Signal-To-Noise Ratio (PSNR) is used to measure deviation of the watermarked and attacked frames from the original video frames and is defined as:

$P S N R=10 \log _{10}\left(255^{2} / M S E\right)$

Where MSE (mean squared error) between the original and distorted frames (size $\mathrm{m} \times \mathrm{n}$ ) is defined as:

$M S E=(1 / m n) \sum_{i=1}^{m} \sum_{j=1}^{n}\left[I(i, j)-I^{\prime}(i, j)\right]$

Where $I$ and $I^{\prime}$ are the pixel values at location (i,j) of the original and the distorted frame respectively. Higher values of PSNR indicate more imperceptibility of watermarking. It is expressed in decibels (dB).

\subsection{Normalized coefficient $(\mathrm{NC})$}

The normalized coefficient (NC) gives a measure of the robustness of watermarking and its peak value is 
$N C=\frac{\sum_{i} \sum_{j} W(i, j) \cdot W^{\prime}(i, j)}{\sqrt{\sum_{i} \sum_{j} W(i, j) \sqrt{\sum_{i} \sum_{j} W^{\prime}\left(i_{s}, j\right)}}}$

Where $W$ and $W^{\prime}$ represent the original and extracted watermark respectively. After extracting and refining the watermark, a similarity measuring of the extracted and therefore the referenced watermarks is used for objective judgment of the extraction fidelity. The performance of algorithm can be measured in terms of its imperceptibility and robustness against the possible attacks. Watermarked frame is subjected to a variety of attacks such as Histogram equalization, Gamma correction, Contrast adjustment, Jpeg compression etc. In case of geometric attacks scheme is tested against Frame rotation, Frame resizing, Frame cropping.

\subsection{Attacks}

"Fig. [1]" shows video sequence 'bus.avi' of speed 30 frame/ second and resolution $352 * 288$. Using for watermark embedding process. watermark image 'Fig. [2] has '1.bmp' of size $100 \times 100$ is embedded with embedding strength $\alpha=30$.

\subsubsection{Contrast Adjustment Attack}

"Figures below $[4,5,6]$ " shows results of contrast adjustment. As contrast adjustment factor increases value of PSNR decreases. When contrast adjustment factor is less good results can be achieved.

Table 1- Values of PSNR after applying contrast adjustment

\begin{tabular}{|l|l|}
\hline Contrast Adjustment(strength) & PSNR \\
\hline 30 & $42.1260 \mathrm{db} \mathrm{NC} \_$contrast adjustment $=0.95$ \\
\hline 40 & $40.8766 \mathrm{db} \mathrm{NC}_{\text {_ contrast adjustment }=0.97}$ \\
\hline 50 & $39.9075 \mathrm{db} \mathrm{NC}$ _ contrast adjustment $=0.98$ \\
\hline
\end{tabular}

4.3.2 Histogram Equalization Attack

"Fig [7]" shows results of Histogram equalization. usually increases the contrast of images.

\subsubsection{Median Filtering Attack}

"Fig . [8]" shows Video frame after Median Filtering attack. The median filter is a nonlinear digital filtering technique used to remove noise.

4.3.4 Geometric Attacks

Here different geometric attacks are performed on video frame like resize and cropping. From results we can see that quality of video frame cannot be changed that is algorithm is robust against different attacks.

\subsubsection{Cropping}

"Figure [9]" shows the effect of geometric attacks carrying out on watermarked video frame. After application of geometric attacks like rotation, resizing, cropping pixel values get changed and value of PSNR decreases.

4.3.6 Salt and paper noise

"Fig. [10]" shows Video frame after addition of salt and paper noise attack. An image containing salt-andpepper noise will have dark pixels in bright regions and bright pixels in dark regions. After application of salt and paper noise pixel values get distorted and hence value of PSNR decreases.

Table 2 Result Analysis

\begin{tabular}{|l|l|}
\hline $\begin{array}{l}\text { ATTACKS For Strength30 } \\
\text { PSNR is 42.1260 }(\mathbf{d b})\end{array}$ & NC Value \\
\hline Contrast Adjustment & 0.958 \\
\hline Histogram Equalization & 0.918 \\
\hline Cropping & 0.984 \\
\hline Median Filtering & 0.971 \\
\hline salt and paper noise & 0.984 \\
\hline Gaussian noise & 0.984 \\
\hline
\end{tabular}

Different attacks performed on watermarked video frame to test robustness and imperceptibility of watermarking. A Peak Signal to Noise Ratio (PSNR) is used as a metric for measuring the distortion in a signal. Thus the PSNR measurement between the original video frame and the watermarked video frame shows that the proposed method maintains the perceptual visual quality of the watermarked frame as close to the original. 
IOSR Journal of Engineering (IOSRJEN)

e-ISSN: 2250-3021, p-ISSN: 2278-8719

Vol. 3, Issue 11 (November. 2013), |VV4|| PP 45-49

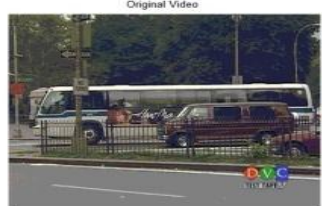

“Fig.1"Original Video Bus.avi 352*288

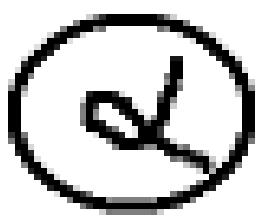

“Fig. 2" Watermark Image1.bmp 100*100

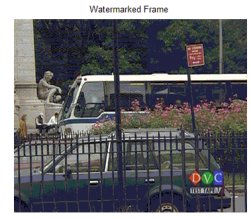

“Fig. 3" Watermarked Video frame.jpg
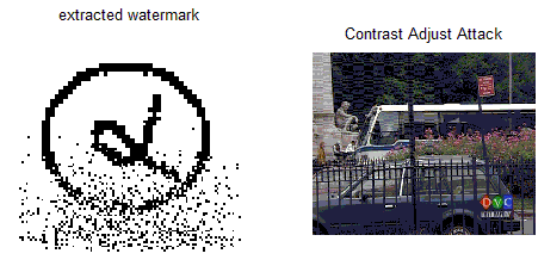

"Fig. 4" Contrast Adjustment by 30

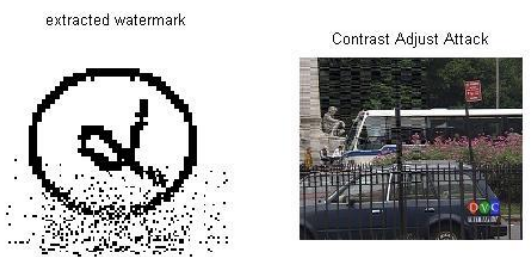

“Fig . 5" Contrast Adjustment by 40

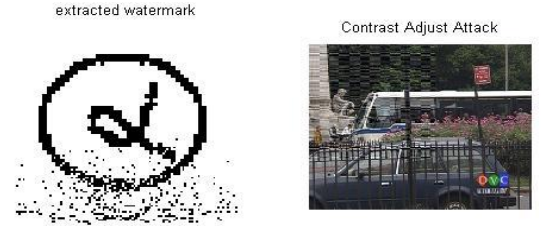

"Fig.6"Contrast Adjustment by 50
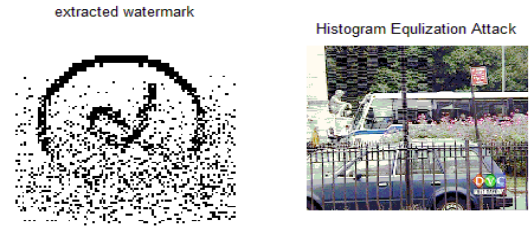

"Fig. 7" Histogram Equalization
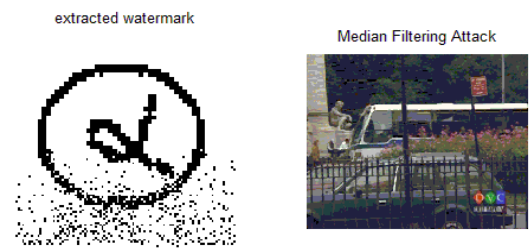

"Fig . 8" Video frame after Median Filtering
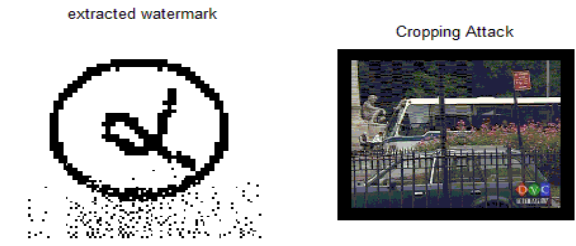

"Fig.9" Video frame after cropping 

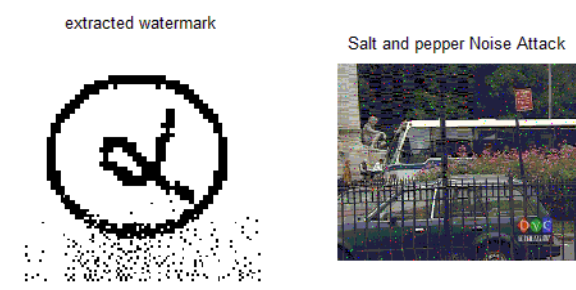

"Fig. 10" Video frame after addition of salt and paper noise

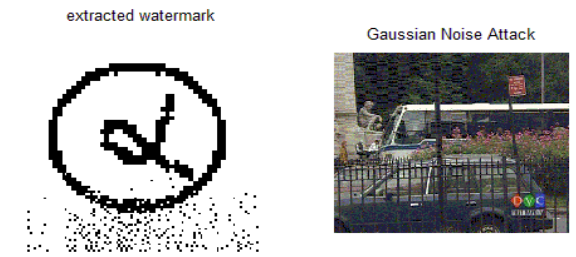

"Fig. 11"Video frame after addition of Gaussian noise

\section{CONCLUSION}

Due to multi resolution characteristics of DWT this scheme is robust against several attacks. It will not affect original quality of video. In this process the PSNR value is same for per attack but NC value will be changed for per attack. PSNR \& NC values are depends on strength. When strength value have minimum then PSNR will be more \& NC will be less. Otherwise When strength value have maximum then PSNR will be less \& NC will be more. The watermark is embedded into the maximum coefficient of the PCA block so we get high imperceptibility where there is no noticeable difference between the watermarked video frames and the original frames.

\section{REFERENCES}

[1] Yeo and M.M. Yeung, "Analysis and synthesis for new digital video applications," icip, International Conference on Image Processing (ICIP'97),vol. 1, pp.1,1997.

[2] M. Natarajan , G. Makhdumi1,"Safeguarding the Digital Contents: Digital Watermarking,” DESIDOC Journal of Library \& Information Technology, Vol. 29, May 2009, pp. 29-35.

[3] C.I. Podilchuk, E.J. Delp "Digital watermarking: algorithms and applications," Signal Processing Magazine, Vol 18,pp. 33-46,IEEE, July 2001.

[4] G. Doërr, J.L. Dugelay, "Security Pitfalls of Frame-by-Frame Approaches to Video Watermarking," Signal Processing, IEEE, Transactions, vol. 52, pp. 2955 - 2964, 2004.

[5] M. K. Thakur, V. Saxena, J. P.Gupta, "A Performance Analysis of Objective Video Quality Metrics for Digital Video Watermarking," Computer Science and Information Technology (ICCSIT), 2010, 3rd IEEE International Conference, Vol. 4, pp. 12 - 17,2010.

[6] S. Voloshynovskiy, S. Pereira, T. Pun, “Watermark attacks,” Erlangen Watermarking Workshop 99, October 1999.

[7] G. Langelaar, I. Setyawan, and R. Lagendijk, "Watermarking Digital Image and Video Data: A State of Art Overview," IEEE,Signal Processing Magazine, vol. , pp. 20-46, Sep. 2000.

[8] F. Hartung and B. Girod, "Watermarking of uncompressed and compressed video," Signal Processing, 1998,vol. 66, no. 3,pp. 283-301.

[9] T. Khatib, A. Haj, L. Rajab, H. Mohammed, “ A Robust Video Watermarking Algorithm”, Journal of Computer Science, vol. 4, 